\title{
Extension des marchés et normalisation : les systèmes agro-alimentaires dans la mondialisation
}

\author{
The agrofood systems within the globalization : \\ between free-trade market extension and the rise \\ of global standards
}

\author{
Annie Lamanthe \\ Laboratoire d'Economie et de Sociologie du Travail - LEST (UMR 6123), \\ 35, avenue Jules Ferry, 13626 Aix-en-Provence cedex.
}

\section{Résumé}

L'article se propose de caractériser le cadre d'action et de contraintes dans lequel les systèmes agroalimentaires ont eu à s'inscrire depuis le début de la décennie 80. Celui-ci est marqué par la «mondialisation » des échanges, entendue ici comme un processus social de construction et d'extension des marchés, via le développement du commerce international. Il met l'accent sur deux aspects de ce processus : la construction de vastes zones de libre-échange, notamment dans le cadre du Mercosur, de l'Union Européenne et de l'OMC ; l'expansion et la multiplication des dispositifs de normalisation.

(C) 2007 Lavoisier, Paris. Tous droits réservés.

\section{Summary}

The article aims to describe some aspects of the globalization context which with agrofood systems have to deal since the beginning of the 80 's. The globalization is seen here as a social process of free-trade market extension at the international level. The article emphasizes two main figures of this process : the building of large free-trade markets areas such as Mercosur and European Union; the rise of global standards.

(c) 2007 Lavoisier, Paris. Tous droits réservés.

*Adresse email : annie.lamanthe@univmed.fr

doi:10.3166/ges.9.257-270 @ 2007 Lavoisier, Paris. Tous droits réservés. 
Mots clés : Systèmes agro-alimentaires ; mondialisation ; normalisation ; commerce international ; construction sociale des marchés.

Keywords: Agrofood system ; globalization ; global standards ; free-trade markets ; economic sociology.

Ce texte se propose de caractériser le cadre d'action et de contraintes dans lequel les systèmes agro-alimentaires ont eu à s'inscrire depuis la décennie 80. Celui-ci est marqué par la «mondialisation » des échanges, que nous entendrons ici comme l'extension croissante des marchés, via le développement du commerce international.

Il n'est pas inutile de rappeler ici que la mondialisation est une construction : la multiplication des zones de libre échange contribue directement à l'intensification des flux de circulation des capitaux et des marchandises. Ces zones sont des dispositifs construits par des acteurs en relation. La mondialisation est orientée : le commerce international se développe aujourd'hui dans une optique fondée sur l'idéologie du libre-échange et sur sa mise en œuvre pratique, optique qui tient notamment aux acteurs qui en sont partie prenante. Les Etats, gouvernements et certaines institutions jouent un rôle prépondérant dans la construction de ces vastes zones de libre-échange et plus largement dans l'avènement des régulations par le marché (Tickell et Peck, 2003 ; Fourcade-Gourinchas et Babb, 2002). L'idéologie du libre-échange prône une ouverture des économies nationales avec l'abandon des protections aux frontières qui doit conduire à de nouveaux équilibres dans lesquels se consolideront les atouts spécifiques de chaque nation. Les produits agricoles et alimentaires n'échappent pas à cette visée, comme le soulignent Boussard et al. (2005) : « libéraliser le commerce des matières premières et faire gérer la production par le marché comme toute autre activité économique est le remède le plus couramment préconisé à l'heure actuelle» (p.7). Pour ces différentes raisons, nous considèrerons ici la mondialisation comme le résultat d'un phénomène de construction sociale du marché, qu'il convient de situer dans les contextes institutionnels dans lesquels il s'opère (Fligstein, 2005).

Le papier veut insister sur cette construction en s'intéressant à deux de ses aspects. En premier lieu, la mise en place de ces vastes zones de libre-échange apparaît comme plus complexe que l'idéologie qui les sous-tend ne semblerait le laisser envisager. En effet, de nombreuses « résistances » se font jour, de la part de pays qui, pour certains, en sont pourtant les plus forts partisans. L'agriculture est au cœur de nombreuses tensions qui révèlent les paradoxes et difficultés de cette construction. En second lieu, il faut évoquer le développement exponentiel de la normalisation, comme corollaire à l'extension des marchés. La normalisation, elle-même construction sociale orientée par des acteurs, apparaît à la fois comme un problème et une nécessité en régime de libre-échange. De fait, le développement du commerce mondial conduit les entreprises des systèmes agro-alimentaires à se situer dans un double mouvement : l'alignement sur des standards internationaux; le respect d'une multiplicité de référentiels normatifs qui conditionnent leur accès au marché et leur maintien sur celui-ci.

\section{Une circulation des produits sur des aires sans cesse élargies}

La circulation de l'orange fraîche entre pays d'Amérique Latine et d'Europe s'inscrit dans le mouvement général d'accélération des échanges de ces vingt-cinq dernières 
années. Ce mouvement est double : il associe développement du commerce international et libéralisation des échanges. Entendu dans le sens du développement des échanges, la mondialisation n'apparaît pas comme un phénomène inéluctable ou spontané mais bien comme un projet et une construction délibérée. Ainsi, les Etats portent largement ce phénomène avec la multiplication des accords bi ou multi-latéraux et la mise en place de blocs régionaux visant à libéraliser les échanges. Les produits agricoles et alimentaires font l'objet d'une insertion croissante dans ce processus. Depuis longtemps et jusqu'il y a peu, l'agriculture s'était trouvée « plus ou moins déconnectée du marché » (Boussard et al., 2005). En effet, elle faisait massivement l'objet de politiques nationales de régulation et de protections douanières vis-à-vis des importations, étant la cible d'un traitement spécifique en tant qu'activité économique. Cette tendance générale s'est récemment inversée sous l'effet d'une volonté d'insérer de façon croissante les produits agricoles dans les échanges mondiaux et de libéraliser ces échanges. Il s'agit ainsi, selon Boussard et al., de «faire gérer la production par le marché comme toute autre activité économique » (p.7). Cette insertion est au cœur de nombreux débats actuels et se pose souvent de façon particulièrement conflictuelle. Ils révèlent notamment les tensions que provoque la mise en concurrence accrue des pays et des producteurs au niveau mondial.

C'est ce mouvement qui est illustré ici en examinant plus particulièrement les principes qui constituent le fondement des dispositifs de développement du libre-échange intervenant dans la circulation de l'orange sud-américaine : le Mercosur, l'Union Européenne (UE) et l'OMC (Organisation Mondiale du Commerce).

\subsection{Mercosur : intégration régionale et ouverture des économies}

C'est le traité d'Asunción, signé en 1991 entre Argentine, Brésil, Paraguay et Uruguay, qui donne naissance au Mercosur. Au départ, le projet est double : favoriser les échanges entre les pays membres et faciliter leur insertion dans le commerce international. Dans ce domaine, il vise à renforcer le processus d'ouverture de chaque pays et son insertion dans les marchés mondiaux, notamment à travers l'implantation d'entreprises étrangères et la captation d'investissements. Il se fonde sur un modèle de développement privilégiant l'ouverture des économies et la stimulation des complémentarités entre nations comme support à leur compétitivité internationale (de la Garza et Salas, 2003).

Le Mercosur fut d'abord une simple zone de libre-échange organisant une libéralisation du commerce entre les quatre pays membres par une réduction progressive des droits de douane (en 2002, 90\% des échanges entre les quatre pays sont exempts de droits de douane) et des « restrictions non tarifaires au commerce » (les normes techniques et sanitaires nationales par exemple). Avec l'instauration de tarifs douaniers extérieurs communs en partie harmonisés, il se transforme en union douanière partielle en 1995 tout en visant à devenir à terme un marché commun régi par une même politique commerciale. Un des groupes techniques créés par le traité d'Asunción est spécifiquement dédié à l'agriculture et à la politique agricole, marquant par là la volonté des pays membres d'avancer vers plus d'intégration dans ce domaine. La réalisation de ce projet a cependant pris du retard par rapport au calendrier et aux ambitions initiales. Les difficultés macro-économiques de ses deux plus importants membres (l'Argentine et le Brésil) en ont été une des causes principales. Des désaccords concernant l'agriculture ont aussi contribué à ce ralentisse- 
ment dans la réalisation des objectifs initiaux ; la protection commerciale des produits agricoles reste élevée au sein même du Mercosur (Mulder et al., 2004).

Cependant, et malgré ce ralentissement, le commerce a augmenté de façon substantielle à la fois entre les pays membres (il a presque triplé) et entre ces pays et le reste du monde (il a presque doublé) au cours des dix années qui ont suivi la création du Mercosur. Au début des années 2000, cette progression s'est ralentie mais le Mercosur représente aujourd'hui une des plus importantes zones de libre-échange au monde. Son effet est aussi tangible en ce qui concerne la capacité des pays qui le composent à attirer les investissements directs étrangers (principalement de l'Europe et de l'Amérique du nord), qui se traduit par l'accroissement de la part de ses pays membres dans l'investissement direct étranger mondial $(5,9 \%)$, notamment favorisé par les « réformes structurelles » internes (privatisations).

\subsection{L'Union Européenne : la construction d'un marché unique et un alignement croissant sur les conditions mondiales}

L'histoire de l'Union Européenne est celle, plus complexe, d'une construction économique, politique et institutionnelle. Le traité de Paris, signé en 1951, avait institué la Ceca (Communauté du charbon et de l'acier) entre six pays (Allemagne, Belgique, France, Italie, Luxembourg et Pays-Bas). La Communauté économique européenne est créée en 1957 par ces six mêmes pays, à l'occasion de la signature du traité de Rome. Celui-ci prévoit l'intégration progressive des économies au sein d'un marché commun. Dix ans après, en 1968, l'union douanière est réalisée (les droits de douane entre pays membres sont supprimés) et un tarif extérieur commun est établi. Anticipé depuis 1986 dans le cadre de l'Acte unique européen, le marché unique se met en place le premier janvier 1993. Il se fonde sur le démantèlement de tous les obstacles à la libre circulation des biens, y compris non tarifaires, et une politique garantissant la libre concurrence.

La politique agricole commune (PAC) est la première politique communautaire ${ }^{1}$, ayant vu le jour avec le traité de Rome lui-même. Au cours du temps, les orientations de cette politique ont favorisé un double mouvement : en interne, la libre circulation progressive des produits agricoles entre pays membres (en organisant des périodes transitoires de protection par des aides et des quotas) jusqu'à l'entrée en vigueur du marché unique en 1993 où quasi toutes les barrières internes ont été supprimées ; vis-à-vis de l'extérieur, un alignement croissant sur les conditions mondiales et une diminution des aides. La PAC est mise en œuvre à partir de 1962, où sont retenus des principes d'action sur le marché visant la liberté complète des échanges au sein des pays membres et l'instauration d'un tarif douanier commun. Le principal instrument de la PAC (les Organisations communes de marché, OCM) vise la régulation des marchés agricoles par une politique des prix et organise la protection des produits vis-à-vis des importations extra-européennes. Au cours des décennies 70 et 80 , différents aménagements vont tenter d'enrayer les problèmes auxquels se trouve très vite confrontée la PAC et qui tiennent à ses principes de base : surproduction, augmentation croissante de ses coûts... A partir de 1992, elle fait l'objet de réformes successives dont l'ob-

\footnotetext{
${ }^{1}$ Les développements qui suivent sur la PAC et son évolution sont principalement tirés de Butault et al., 2004.
} 
jectif est de réduire sa part dans le budget européen mais qui s'inscrivent aussi assez directement dans une orientation de libéralisation croissante des échanges au niveau international. La tendance de ces différentes réformes $(1992,1999,2003)$ est de favoriser un alignement des prix sur les cours mondiaux, avec une diminution continue des prix d'intervention et la modification des systèmes d'aides, qui tendent à être de plus en plus déconnectés des productions. C'est aussi le système d'une agriculture productiviste que la PAC a elle-même fait advenir qui est remis en cause, avec le souci plus grand de l'environnement et de la santé des consommateurs. Ces réformes sont aussi très articulées aux négociations internationales et aux pressions nord-américaines (l'agriculture est introduite dans les négociations de l'Organisation Mondiale du Commerce en 1994).

L'Union Européenne est le principal partenaire du Mercosur pour le commerce et les investissements (elle représente $30 \%$ des courants d'échange de celui-ci). Les produits agricoles et agro-alimentaires représentent $50 \%$ des exportations totales du Mercosur vers l'Union Européenne (principalement : oléagineux et produits dérivés ; fruits et produits dérivés ; café, thé ; cacao et épices). Dans l'autre sens, leur part ne s'élève qu'à $4 \%$ du total (principalement : boissons, céréales et produits dérivés, végétaux et produits dérivés, produits laitiers et œufs) (Mulder et al.). Depuis le début des années 90, les exportations et les investissements de l'UE vers le Mercosur se sont intensifiés². En 1995, les deux blocs ont signé un accord de coopération et formé le projet de constituer une zone de libre échange qui prévoit une libéralisation réciproque et progressive des échanges agricoles. Les négociations ont débuté en 2000 mais viennent cependant buter sur la question des subventions agricoles de l'Union Européenne, « dénoncées » auprès de l'OMC par certains des pays membres du Mercosur.

\subsection{L'Organisation mondiale du commerce : \\ l'intégration de l'agriculture dans le marché}

L'OMC est créée le premier janvier 1995. Cette organisation découle de négociations conduites au niveau international depuis 1986 dans le cadre de ce qu'on appelle l' «Uruguay round ». Elle succède au GATT (General agreement on tariffs and trade), qui date de 1948. Ce dernier visait principalement le commerce des marchandises. L'OMC correspond à un élargissement du champ de compétence de la négociation internationale qui inclut dès lors les services et les échanges d'inventions, de créations et de dessins et modèles (sous-tendus par l'idée de propriété intellectuelle). En février 2006, l'OMC compte 149 pays membres.

L'OMC se présente elle-même comme « un cadre de négociation, un ensemble de règles et un dispositif pour le règlement des différends qui ont trait au commerce international » s'appliquant à l'ensemble de ses membres. Les principes qui en sont au fondement sont les suivants : « un commerce sans discrimination », «l'égalité de traitement des produits nationaux et des produits étrangers »; « une libéralisation progressive du commerce et

${ }^{2}$ Le Mercosur n'a pas été, au départ, un cadre réellement pertinent pour le commerce des agrumes (il est très peu mentionné par les entreprises rencontrées dans la recherche). Par ailleurs, il a conduit à une formalisation de relations qui existaient déjà entre pays (Uruguay et Argentine), ce qui en a fait des contraintes et a eu tendance à réduire ces échanges plutôt qu'à les développer. L'intensification des échanges avec l'UE peut se comprendre aussi comme un effet de cette situation. 
par voie de négociation »; « la prévisibilité du commerce grâce à la consolidation et la transparence »; « la promotion et la garantie d'une concurrence loyale »; « les réformes économiques et une contribution au développement»(OMC). Cette instance promeut notamment la libéralisation des échanges comme gage de développement et de progrès : «... des politiques commerciales libérales -celles qui garantissent la circulation sans restriction des biens et des services - accroissent la concurrence, encouragent l'innovation et engendrent le succès. Elles amplifient le bénéfice que l'on peut retirer de la production la meilleure, la mieux conçue et effectuée au meilleur prix » (OMC).

Jusqu'en 1994, l'agriculture a bénéficié d'un statut particulier dans les négociations internationales. L'Union européenne avait en effet obtenu, contre les Etats-Unis, que l'agriculture soit traitée, dans ce mouvement de libéralisation croissante des échanges internationaux, d'une façon différenciée de celle dont l'étaient les autres secteurs marchands (Butault et al., 2004). Jusqu'alors, le GATT couvrait les produits agricoles mais autorisait une certaine «exception agricole»: les pays avaient en effet la possibilité de maintenir des droits de douane, de contingenter leurs importations et de verser des subventions à leurs producteurs. L'accord sur l'agriculture signé dans le cadre de l' « Uruguay round » met un terme à cette exception : il vise à réformer le commerce dans ce secteur et à renforcer le rôle du marché dans l'orientation des politiques le concernant. Cet accord prévoit notamment l'engagement des signataires à ouvrir leur marché aux produits agricoles et alimentaires venant de l'extérieur, à réduire leur soutien interne aux producteurs et à limiter le subventionnement de leurs exportations. Pour Butault et al., ce processus traduit le fait que « les Etats admettent ainsi que leur politique agricole ne dépend pas seulement de leur propre ressort mais qu'elle doit être soumise à une certaine discipline selon les règles définies au niveau international » (p.122).

Ce mouvement général de multiplication des zones de libre-échange favorise et organise la circulation des biens sur des aires géographiques sans cesse élargies. Qu'il s'agisse de constituer de vastes marchés intégrés à l'échelle d'un continent ou d'organiser une circulation mondiale des produits, le marché unique européen, le Mercosur ou encore l'OMC contiennent cette même visée. Pour les systèmes agro-alimentaires, ce mouvement se traduit par le développement des opportunités de commercialisation. Dans le même temps, il contribue à un élargissement des aires de mise en concurrence, - intrazone ou inter-zones-, de pays où les conditions de production sont déséquilibrées, soit au plan institutionnel (existence de subventions à l'agriculture dans certains pays), soit au plan économique (différentiels dans les coûts de production).

\section{La normalisation au cour du commerce international}

A la fois conséquence du développement du commerce international et support à ce développement, la normalisation des produits est au cœur du mouvement d'extension des marchés. La question de la normalisation s'y pose d'un double point de vue. D'une part, on observe qu'elle s'inscrit au carrefour d'un ensemble complexe et contradictoire d'objectifs et d'impératifs : santé publique versus respect de la liberté du commerce et, dans ce sens, elle apparaît tout à la fois comme une nécessité et comme un problème. De l'autre, elle connaît un développement exponentiel : le champ de la normalisation ne cesse de s'élargir alors que, dans le même temps, se multiplient les référentiels circulant 
au niveau international et qu'apparaissent de nouveaux acteurs de la normalisation. Ceci nous conduit à souligner le caractère quelque peu paradoxal du développement de la normalisation en régime de libre-échange avec, d'un côté, une tendance à limiter la diversité des systèmes nationaux au profit d'un alignement sur quelques standards internationaux avec l'argument central des risques d'entrave à la libre concurrence et, de l'autre, la multiplication des référentiels portés par des accords privés ou semi-privés.

\subsection{La normalisation, à la fois nécessité et problème dans le commerce international}

Dans le commerce international, la normalisation apparaît comme une nécessité. En effet, les normes informent sur les caractéristiques des produits et permettent la large reconnaissance de leur qualité, condition nécessaire quand ces produits circulent sur des marchés élargis (Eymard-Duvernay, 1986). C'est également une nécessité car, s'agissant de produits destinés à la consommation humaine, se pose la question de la santé et de la sécurité alimentaire face aux risques inhérents à la distribution de masse. A l'inverse, une des préoccupations majeures des tenants du libre-échange est d'éviter que les normes techniques et autres réglementations sanitaires dont chaque pays est susceptible de se doter ne viennent jouer comme des obstacles au commerce et fausser la libre concurrence. De fait, la diversité des systèmes nationaux est un problème pour le commerce international dont le développement repose sur la généralisation des standards internationaux. Toutes les initiatives en matière de libre échange sont confrontées à cette problématique d'ensemble. Les travaux conduits au sein de l'OMC et à l'occasion de la construction du Mercosur en sont de bonnes illustrations.

\subsubsection{OMC : entre liberté des pays et alignement sur les normes internationales}

L'accord sur l'agriculture signé en 1994 dans le cadre de l'OMC a contribué à une réduction notable des restrictions quantitatives à la circulation des produits agricoles et alimentaires (selon l'OMC, les droits de douane auraient été réduits de $40 \%$ depuis 1995). Dans le même temps, les normes réglementaires, d'ordres sanitaire et phytosanitaire, adoptées par chaque pays pour protéger la santé animale et humaine ont été considérées comme pouvant constituer un obstacle de type technique au commerce. L'article 20 de cet accord «permet aux gouvernements d'intervenir dans les échanges à des fins de protection de la santé et de la vie des personnes et des animaux ou de préservation des végétaux, à condition qu'ils n'établissent pas de discrimination et qu'ils n'usent pas de cette intervention comme protectionnisme déguisé » (OMC).

L'OMC a fixé un certain nombre de règles en la matière, à l'occasion de deux accords qui, selon Bourif et Pineiro (2000), « fixent les conditions de transparence et d'égalité de traitement pour l'application des mesures réglementaires ». Ces accords tendent à concilier la liberté des pays d'édicter leurs propres normes et les impératifs de la liberté du commerce. Ils posent donc un ensemble de conditions à cette latitude nationale.

Le premier, qui porte de façon générale sur les « obstacles techniques au commerce » (accord OTC), concerne les règlements techniques, normes industrielles, procédures d'essai et d'homologation. Il se fonde sur deux principes : la reconnaissance du droit des Etats à adopter les normes qu'ils jugent appropriées et les mesures nécessaires pour les faire respecter ; un code de conduite qui doit servir dans les processus d'élaboration, 
d'adoption et d'application des normes nationales. Dans le même sens, les procédures d'évaluation de la conformité des produits à ces normes doivent être jugées justes et équitables (OMC). Le second de ces accords a trait plus directement aux produits agricoles et alimentaires et notamment à tout ce qui concerne les normes sanitaires (accord sur les mesures sanitaires et phytosanitaires ou SPS). Dans ce domaine aussi, les pays membres sont autorisés à établir leurs propres normes mais doivent respecter certaines conditions : l'adoption de réglementations plus élevées que les normes internationales doit s'accompagner d'une justification scientifique (notamment fondée sur une évaluation des risques pour la santé et sur le principe de précaution), d'une part ; toutes les mesures sanitaires et phytosanitaires, pour les produits qui concernent le commerce international, doivent être élaborées et appliquées conformément aux dispositions de cet accord, de l'autre. Cet accord s'intéresse aussi aux procédures de contrôle, d'inspection et d'homologation : «Les pays sont autorisés à appliquer des normes différentes d'inspection des produits, sous la condition de pouvoir démontrer que les mesures qu'ils appliquent pour ces exportations assurent le même niveau de protection sanitaire que dans le pays importateur, ce dernier étant alors censé accepter les normes et méthodes de l'exportateur » (OMC).

Au-delà de la question des obstacles techniques au commerce, c'est en définitive la diversité des normes et des pratiques découlant de la multiplicité des systèmes nationaux qui pose problème au commerce international. D'où l'appel réitéré à un alignement sur les normes internationales : «Les règlements techniques et les normes industrielles jouent un rôle important, mais ils varient d'un pays à l'autre. L'existence d'un si grand nombre de normes différentes rend les choses difficiles pour les producteurs et les exportateurs. Si les normes sont fixées arbitrairement, elles pourraient servir de prétexte au protectionnisme. Les normes peuvent devenir des obstacles au commerce », «Afin d'éviter une trop grande disparité, l'accord encourage les pays à appliquer les normes internationales lorsque celles-ci sont appropriées », «Afin d'harmoniser le plus largement possible les mesures sanitaires et phytosanitaires, les Membres établiront les mesures sanitaires ou phytosanitaires sur la base de normes, directives ou recommandations internationales, dans le cas où il en existe »... (OMC).

En ce qui concerne l'agriculture et l'alimentation, trois principales sources de normalisation sont reconnues en tant que références internationales auxquelles tous les pays sont renvoyés. Les normes du Codex Alimentarus sont la référence pour les produits alimentaires. Elles sont élaborées par une commission émanant de la FAO et de l'OMS, créée en 1963. Les objectifs en sont à la fois : la protection de la santé des consommateurs, la promotion de pratiques loyales dans le commerce des aliments, la coordination de tous les travaux de normalisation (Codex Alimentarus). Les normes de la Convention internationale pour la protection des végétaux (CIPV) font référence en matière de préservation des végétaux (leur champ d'action s'étend des plantes cultivées à la flore naturelle, de la terre aux moyens de transport, conteneurs, lieux de stockage...). Créée en 1951 (auprès de la FAO et de l'ONU), la CIPV compte 139 pays membres. Son objectif « est d'assurer une action commune et efficace afin de prévenir la dissémination et l'introduction d'organismes nuisibles aux végétaux et produits végétaux, et de promouvoir des mesures appropriées pour leur contrôle ». De même, elle constitue « un cadre et un forum pour la coopération internationale, l'harmonisation et les échanges techniques entre les parties contractantes » (CIPV)... En ce qui concerne les animaux, les normes de l'organisation mondiale de la santé animale (OIE) sont la référence. 


\subsubsection{La problématique de la normalisation dans la construction du Mercosur}

Dans le cadre de la construction du Mercosur, la nécessité de se livrer à un travail d'harmonisation des normes techniques est posée dès la signature du traité d'Asuncion en 1991. Cette nécessité apparaît comme condition à la réalisation de l'union douanière dans la perspective d'éviter les distorsions à la libre circulation des produits entre les pays partie prenante. Le processus d'harmonisation est considéré comme une des actions à mettre prioritairement en œuvre pour réaliser les objectifs du traité. Chaque pays possède alors son propre système de normes techniques. Il s'agit tout à la fois de « déréguler » ce qui relève du niveau national (disparition des normes nationales) et « d'harmoniser » au niveau du bloc régional (source Mercosur).

A la fin de 1991, dans le cadre de la mise en place des groupes techniques du Mercosur, le groupe $n^{\circ} 3$ (Normes techniques) a en charge l'harmonisation des normes. Un Comité Mercosur de normalisation (normalisation volontaire) se met en place, intégrant les différentes associations nationales de normalisation. Il ne concerne pas l'agriculture. Pour les produits alimentaires, ce travail est réalisé dans le cadre d'une commission spécifique (Comisión de alimentos y metrología legal) de ce groupe $n^{\circ} 3$ et en relation étroite avec le groupe $n^{\circ} 8$ (Agriculture) $)^{3}$. Il aborde une variété de thèmes : information entre pays membres sur les normes techniques; ingrédients et additifs alimentaires ; dénomination commerciale, identité et qualité ; conditionnement ; contaminants... Ce travail aboutit, à la fin de 1994, à l'adoption d'un certain nombre de décisions et de résolutions du Conseil du Marché Commun et du Groupe Marché Commun du Mercosur (instances décisionnelles du Mercosur). Ces décisions sont considérées comme devant servir de cadre légal d'action en matière alimentaire pour les quatre pays membres. Ces derniers doivent à la fois incorporer ces résolutions dans leur législation, promouvoir leur application et poursuivre l'effort d'harmonisation et d'actualisation, prévue pour se dérouler de façon permanente.

Assis de Almeida (2005) montre bien, d'un point de juridique et d'une façon plus large que pour les seules normes techniques, que ce travail d'harmonisation conduit à mettre en tension les normes nationales existantes et les nouvelles normes régionales qui doivent être intégrées dans le droit de chaque pays. Se pose ainsi la question de savoir quelle norme fait valeur, à quel niveau et de quelle façon. Dans le même temps, se trouve aussi directement posée la question de l'articulation des normes régionales avec les standards internationaux. Ce travail d'harmonisation réalisé au sein du Mercosur est aussi une mise en rapport avec ces derniers.

Le sous-groupe $\mathrm{n}^{\circ} 3$ étudie ainsi la possibilité de s'appuyer sur les normes internationales (Codex Alimentarus) pour élaborer les normes régionales. Différents statuts sont donnés aux normes internationales : elles peuvent être adoptées en l'état, elles peuvent servir de base de discussion et faire l'objet d'adaptations tenant compte des spécificités locales. Dans cette opération, le sous-groupe bénéficie d'une aide technique de la FAO en matière d'élaboration des procédures de normalisation, d'inspection et de certification harmonisées. Cette collaboration est aussi l'occasion d'une sensibilisation des professionnels et des chefs d'entreprises agro-alimentaires à l'importance du respect des normes internationales et des accords signés dans le cadre de l'OMC comme gage d'une insertion « réussie » dans le commerce mondial (Costarrica et Dawson).

\footnotetext{
${ }^{3}$ Cette partie s'appuie principalement sur Costarrica et Dawson (1995)
} 


\subsection{Elargissement du champ de la normalisation et montée des acteurs privés}

L'expansion du commerce international au cours des années 1990 a eu deux autres principales implications en matière de normalisation. En premier lieu, cette expansion s'est traduite par un élargissement constant du champ de la normalisation. Ainsi, comme l'observe Noiville (2003) « ... l'aliment suscite une harmonisation juridique croissante. Son emballage, son étiquetage, l'évaluation de sa sécurité, sont désormais strictement encadrés par le droit de l'OMC, non seulement pour empêcher les pratiques frauduleuses et protéger la santé des consommateurs mais aussi pour faciliter le commerce et prévenir les entraves à la circulation de ces produits qui, en vertu du principe de libre-échange, ont vocation à circuler librement sur le marché mondial » (p.64). En second lieu, on observe une multiplication des référentiels circulant au niveau international, qui s'est accompagnée de la montée de nouveaux acteurs de la normalisation (Graz, 2004). Ce processus traduit le phénomène décrit par Messner (2005) d'insertion croissante des entreprises dans des chaînes de valeur globales développant leurs propres formes de « gouvernance » et dans des standards élaborés, gérés et sanctionnés par des réseaux, privés ou privéspublics, agissant eux-mêmes au niveau international.

L'évolution du travail de normalisation au sein de l'Union Européenne et celle des orientations qui lui ont été successivement données permettent d'éclairer ces nouvelles facettes de la normalisation.

\subsubsection{UE : évolution des choix et élargissement du champ de la normalisation}

La normalisation européenne a d'abord concerné les caractéristiques physiques des produits agricoles afin d'en faciliter la circulation. Dès 1972, la CEE instaure pour les fruits et légumes frais une normalisation obligatoire portant sur leurs caractéristiques physiques (règlement CEE 1035/72) dans le but « de favoriser le fonctionnement et le développement du marché, en garantissant au consommateur la qualité de ce qu'il achète et en permettant au producteur de faire valoir la qualité de ce qu'il produit » et « de permettre des échanges commodes entre les différents Etats membres ». Les denrées circulant au sein des pays européens concernés doivent s'y conformer, ainsi que les produits européens exportés hors d'Europe comme les fruits et légumes produits à l'extérieur destinés à être importés.

A la fin des années 80, la Commission européenne renonce au principe d'une normalisation de l'ensemble des produits alimentaires transformés amenés à circuler sur le marché intérieur (à l'inverse de ce qui avait donc été fait pour les fruits et légumes frais). Elle encourage toutefois les professionnels, aidés des Etats membres, à élaborer eux-mêmes une normalisation de leurs produits. Elle modifie ainsi l'orientation du travail de normalisation : en donnant plus de latitude aux professionnels, en insistant sur le caractère volontaire de l'adoption par les entreprises des normes ainsi produites. Adoptant ainsi une «nouvelle approche » selon ses propres termes, la Commission européenne limite son rôle à la seule élaboration des dispositions réglementaires générales. Dans ce domaine, à partir du début des années 90, la Commission va élaborer une série de directives et de règlements qui vont élargir de façon croissante le champ de la réglementation et de la normalisation pour s'intéresser, en plus des caractéristiques des produits, aux méthodes de production et aux modes d'organisation. 
L'hygiène, la sécurité alimentaire, et leur contrôle, sont au cœur de cette nouvelle approche, comme étant essentiels «au bon fonctionnement du marché » (directive 93/43/CEE sur l'hygiène des denrées alimentaires). Elle agit sur plusieurs registres. Premièrement, elle établit les règles essentielles d'hygiène nécessaires à la libre circulation des denrées alimentaires : les entreprises du secteur alimentaire doivent respecter la réglementation lors de la préparation, la transformation, la fabrication, le conditionnement, le stockage, le transport et la distribution, la manutention et la vente ou la mise à disposition des denrées alimentaires. Elle couvre donc ainsi toute la chaîne. Elle stipule que les entreprises doivent assurer l'analyse des risques en leur sein à l'aide de la méthode HACCP (Hazard Analysis Critical Control Points traduit par Analyse des dangers, points critiques pour leur maîtrise), dont l'adoption devient obligatoire. Par ailleurs, elle recommande le recours aux normes ISO 9000 relatives à la qualité et aux systèmes d'assurance de la qualité, - qui sont un moyen d'assurer le respect des règles générales d'hygiène alimentaire -, et l'encouragement par les Etats membres à l'élaboration, par les milieux professionnels, de guides de bonne pratique d'hygiène, souvent fondés eux-mêmes sur l'HACCP (DGCCRF, novembre 1999, « le point sur la transposition de la directive 93/43/CE dans le domaine des produits végétaux »). Les directives 89/397/CEE et 93/999/CE définissent les principes généraux régissant les contrôles officiels des denrées alimentaires. La seconde introduit l'obligation d'une mise aux normes des organismes de contrôle eux-mêmes.

Les différentes crises alimentaires qui ont suivi ont cependant mis en évidence les « faiblesses dans la conception et l'application la réglementation alimentaire » (peu de cohérence, de transparence) qui ont conduit le niveau européen à engager une nouvelle réflexion à son propos (Livre blanc sur la sécurité alimentaire, janvier 2000) et à définir une législation de l'alimentation. Elle aboutit au règlement CE/178/2002 «établissant les principes généraux et les prescriptions générales de la législation alimentaire, instituant l'Autorité européenne de sécurité des aliments et fixant des procédures relatives à la sécurité des denrées alimentaires ». Ce règlement réitère le constat selon lequel la diversité des législations concernant la circulation des denrées alimentaires entre les Etats membres est susceptible « d'entraver la libre circulation des denrées alimentaires, de créer des inégalités en matière de concurrence et, de ce fait, d'influer directement sur le fonctionnement du marché intérieur » et propose de rapprocher certains éléments « de manière à ce qu'ils forment une base commune pour les mesures régissant les denrées alimentaires et les aliments pour animaux adoptés dans les Etats membres et au niveau communautaire ». Ce règlement stipule une approche globale et intégrée de la sécurité alimentaire, la législation concernant aussi les produits exportés, importés, importés et ré-exportés. Cette approche couvre « tous les aspects de la chaîne de production alimentaire dans sa continuité, à partir de la production primaire et de la production d'aliments pour animaux et jusqu'à la vente ou à la fourniture de denrées alimentaires au consommateur ». Le règlement rend obligatoire, à échéance 2005, leur traçabilité complète, celle-ci étant définie comme la «capacité de retracer, à travers toutes les étapes de la production, de la transformation et de la distribution, le cheminement d'une denrée alimentaire, d'un aliment pour animaux, d'un animal producteur de denrées alimentaires ou d'une substance destinée à être incorporée ou susceptible d'être incorporée dans une denrée ou un aliment pour animaux » (UE). 


\subsubsection{La montée de référentiels privés}

Dans le même temps que le niveau européen décide de privilégier, au-delà des aspects réglementaires, une normalisation à caractère volontaire et d'accorder aux acteurs professionnels un rôle central dans l'élaboration de normes, la distribution devient un acteur puissant de ce processus. La décennie 90 voit en effet se multiplier les référentiels que les distributeurs élaborent à destination de leurs fournisseurs au niveau international. Ces référentiels ne concernent plus seulement les caractéristiques de produits mais couvrent l'ensemble des processus de production. On présentera ici les plus importants.

La certification EUREPGAP est créée à l'initiative de la grande distribution nord européenne en 1997, en direction des producteurs agricoles. C'est une certification internationale qui est censée pouvoir s'appliquer à l'échelle mondiale. Elle suppose le suivi, contrôlé par des audits successifs réalisés par des organismes agréés, d'un référentiel normatif auquel le producteur doit se conformer. Elle conditionne de plus en plus l'accès aux marchés nord-européens tenus par ces grands distributeurs. L'objectif affiché par les concepteurs est explicitement de répondre aux préoccupations des consommateurs sur plusieurs registres : la santé alimentaire, le bien-être animal, la protection de l'environnement et l'amélioration générale des conditions de travail. Il s'agit précisément, dans ce cas, d'une intervention directe des distributeurs sur les modes de production agricoles, l'idée étant d'établir, avec ce référentiel « un cadre de Bonnes pratiques agricoles (ou GAP, abréviation de l'anglais 'Good Agricultural Practice') définissant les éléments essentiels pour le développement des meilleures pratiques dans la production mondiale de produits agricoles. Il définit la norme minimale acceptable pour les grandes enseignes de la grande distribution en Europe ». La certification suppose la mise en place d'une traçabilité complète des produits, des pratiques de «lutte intégrée » et de « gestion intégrée des cultures » et encourage l'application des principes de l'HACCP (Document EUREPGAP, Référentiel EUREPGAP pour fruits et légumes frais, version : septembre 2001, traduction française).

Deux référentiels, celui du BRC (British Retail Consortium Food Technical Standard) et l'IFS (International Food Standard), émanent de regroupements de distributeurs et s'adressent à leurs fournisseurs de produits alimentaires transformés. Le premier, publié en 1998 pour répondre à la directive européenne et aux exigences du gouvernement britannique sur la sécurité alimentaire, émane d'un regroupement des distributeurs du Royaume-Uni. Le second a été créé en 2002 par une organisation de distributeurs allemands et a été repris en 2003 par les distributeurs français via la Fédération des entreprises du commerce et de la distribution. Tous deux ont comme préoccupation majeure la sécurité alimentaire. Le second concerne plus particulièrement les produits fabriqués pour être vendus sous la marque du distributeur : «l'IFS a été conçu, en tant qu'outil commun, pour assurer la sécurité alimentaire et pour surveiller le niveau qualitatif des fabricants de produits à marque de distributeur. Le référentiel est applicable à toutes les étapes de fabrication des produits alimentaires, ultérieures à la production agricole ». Il formule des exigences dans les domaines suivants : « gestion du système qualité (exigences relatives à l'HACCP, manuel qualité, obligations d'enregistrement), « responsabilité du management » (responsabilités et engagements de la direction, vérification des systèmes de qualité et de production pour optimiser les résultats), « gestion des ressources » (exigences concernant le personnel, l'hygiène, les locaux et les équipements), « processus 
de production » (liste détaillée des exigences concernant les diverses étapes de fabrication du produit : locaux, équipements, hygiène, traçabilité, gestion des déchets...), « mesures, analyses, amélioration » (exigences concernant la température, le temps et la qualité, la maîtrise des contaminations...) (source IFS). Dans les deux cas, il s'agit d'une opération de rationalisation et de standardisation du système de référencement et d'évaluation des fournisseurs par leurs clients, les distributeurs. L'objectif est de réaliser des économies d'échelle en adoptant un standard commun unique et permettant une reconnaissance mutuelle des fournisseurs par les distributeurs, alors qu'auparavant chaque distributeur pouvait développer son propre système.

\section{Conclusion}

D’une façon générale, dans le processus d'extension des marchés, la normalisation se déploie à plusieurs niveaux et sert des objectifs multiples. Elle relève de la réglementation en matière d'hygiène de sécurité alimentaire qui, désormais, concerne directement les processus de production et les méthodes de travail (avec le développement de méthodes standard comme l'HACCP par exemple). Elle relève d'un mode d'organisation des relations entre clients et fournisseurs qui renchérissent souvent sur les standards internationaux en matière de sécurité alimentaire et de protection de l'environnement et, dans certains cas, de conditions de travail afin de satisfaire aux préoccupations des consommateurs dans ces domaines (les référentiels des grands distributeurs par exemple). Enfin, elles s'inscrit dans des stratégies commerciales de distinction avec la création de labels, de marques... qui supposent le respect de cahiers des charges précis (Appellations d'origine contrôlées - AOC, Inscriptions géographiques de provenance - IGP, marques privées...). Pour les entreprises des systèmes agro-alimentaires, le respect de plusieurs niveaux de référentiels qui peuvent s'emboîter ou se juxtaposer apparaît aujourd'hui comme une des conditions à satisfaire pour entrer et se maintenir sur le marché dans le contexte de la mondialisation.

\section{Références}

Assis de Almeida J.-G., 2005, Le cadre normatif du Mercosur, Droit et Société, 59, 39-55

Bourif E., Pineiro M., 2000, Nouvel environnement du commerce international et pays en développement : l'impact des accords SPS et OTC, actes de l'atelier international Cirad-Fao, Montpellier, 11-13 décembre

Boussard J.-M., Gérard F., Picketty M.-G., 2005, Libéraliser l'agriculture mondiale? Théories, modèles et réalités, Montpellier, Cirad

Butault J.-P., Gohin A., Guyomard H., 2004, Des repères historiques sur l'évolution de la politique agricole commune, in Butault J.-P. (ed.), Les soutiens à l'agriculture. Théorie, histoire, mesure, Paris, Inra Editions, 85-118

Costarrica M.-L., Dawson R. 1995, La normalización de alimentos y el Codex Alimentarus en el marco del Mercosur, Food, Nutrition and Agriculture, 15 (www.fao.org/docrep/V9723T/v9723t00.htm)

De la Garza Toledo E., Salas C. (comp.), 2003, Nafta y Mercosur. Procesos de apertura economica y trabajo, Buenos Aires, Clacso 
Eymard-Duvernay F., 1986, La qualification des produits, in Salais R., Thévenot L. (eds), Travail : marchés, règles, conventions, Paris, Economica, 239-247

FAO, 2001, Les principales tendances du commerce international et du commerce des produits agricoles (www.fao.org)

Fligstein N., 2005, States, Markets and Economic Growth, in Nee Victor, Swedberg Richard (ed), The Economic Sociology of Capitalism, Princeton University Press, 120-143

Fourcade-Gourinchas M., Babb S. L., 2002, The Rebirth of the Liberal Creed : Paths to Neoliberalism in Four Countries, American Journal of Sociology, vol.108, 3, 533-579

Graz J.-C., 2004, Quand les normes font loi : topologie intégrée et processus différenciés de la normalisation internationale, Etudes Internationales, XXXV, 2, 233-260

Messner D., 2005, Regions in the « world economic triangle », in Schmitz Hubert (ed), Local Enterprises in the Global Economy. Issues of Governance and Upgrading, Edward Elgar, 20-52

Mulder N., Vialou A., David B., Rodriguez M., Castilho M., 2004, La compétitivité de l'agriculture et des industries agroalimentaires dans le Mercosur et l'Union Européenne dans une perspective de libéralisation commerciale, CEPII, Document de travail, 19 novembre

Noiville C., 2003, Brèves réflexions sur la reconnaissance d'un «droit à la différence alimentaire » dans le commerce international, Sociologie du Travail, vol.45, 1, 63-76

Tickell A., Peck J., 2003, Making global rules, in Peck J. and Yeung H. (eds), Global connections, London, Sage

\section{Sites Internet :}

BRC, www.brc.org

CIPV, www.ippc.int

Codex Alimentarus, www.codexalimentarus.net

IFS, International Food Standard www.food-care.info

Mercosur (Guia del Mercosur), www.enapro.com.ar

Organisation mondiale du commerce (OMC), www.wto.org

Union Européenne (UE), www.info-europe.fr 